\title{
Outflow sources in the Vela region ${ }^{\star}$
}

\author{
J.G.A. Wouterloot ${ }^{1}$ and J. Brand ${ }^{2}$ \\ 1 Radioastronomisches Institut, Universität Bonn, Auf dem Hügel 71, D-53121 Bonn, Germany \\ e-mail: wouterloot@astro.uni-bonn.de \\ 2 Istituto di Radioastronomia, CNR, Via Gobetti 101, I-40129 Bologna, Italy \\ e-mail: brand@terra.bo.cnr.it
}

Received July 9; accepted August 25, 1999

\begin{abstract}
We have observed ${ }^{12} \mathrm{CO}(1-0),{ }^{13} \mathrm{CO}(1-0)$, $\mathrm{C}^{18} \mathrm{O}(1-0)$, and $\mathrm{CS}(2-1)$ towards nine sources in the Vela region for which the ${ }^{12} \mathrm{CO}(1-0)$ spectra by Wouterloot \& Brand (1989) suggested the presence of strong line wings (towards two of those sources only $\mathrm{CS}$ or ${ }^{12} \mathrm{CO}$ and ${ }^{13} \mathrm{CO}$ were observed). The sources are located in or behind the Vela Molecular Ridge at distances between about 0.6 and $6.7 \mathrm{kpc}$. In all transitions we made small maps, typically several arcminutes in size.

Towards five sources the ${ }^{12} \mathrm{CO}$ emission is confused by unrelated components at other velocities (not visible in CS and $\mathrm{C}^{18} \mathrm{O}$ ) which we subtracted from the line profiles before analysing the wing emission. The presence of outflow emission was studied by also subtracting the contribution of a central Gaussian line component. We conclude that of the eight sources observed in $\mathrm{CO}$, seven show outflows, one does not. For one of the sources only the blue wing could be studied due to the confusing presence of other strong line components. For the outflow sources we derive the physical parameters. Wing emission has a relative outflow velocity up to about $16 \mathrm{~km} \mathrm{~s}^{-1}$, but the average (weighted with line intensity) is about $4 \mathrm{~km} \mathrm{~s}^{-1}$. Masses of the outflows range from 1 to $150 M_{\odot}$.

$\mathrm{CS}$ and $\mathrm{C}^{18} \mathrm{O}$ were detected towards all eight (resp. seven) sources observed in these molecules. Five sources show single clumps in both transitions, two sources show several clumps in one of the transitions (the other was not mapped completely) and one source shows only weak emission (probably due to its distance). Clump masses and radii range from several $10 M_{\odot}$ to about $1000 M_{\odot}$ and 0.2 pc to 2 pc. Five of the sources show a velocity gradient in $\mathrm{CS}$ and $\mathrm{C}^{18} \mathrm{O}$ indicative of rotation.
\end{abstract}

Key words: ISM: clouds — molecules — radio lines: ISM

\footnotetext{
Send offprint requests to: J.G.A. Wouterloot

* Based on observations collected at the European Southern Observatory, Chile.
}

\section{Introduction}

In molecular clouds there are regions of energetic mass outflow, that are restricted to a small area of a cloud, usually surrounding an embedded young stellar object (YSO; see e.g. Lada 1985; Bachiller 1996). The typical signature of these outflows are broad (up to $\approx 20 \mathrm{~km} \mathrm{~s}^{-1}$ for "standard high-velocity" flows; up to $150 \mathrm{~km} \mathrm{~s}^{-1}$ for the weak, "extremely high-velocity" flows), low-level emission superimposed on a narrow emission line. The broad component is often distributed in a bipolar fashion. As these flows are much more often associated with embedded IR sources than with optically visible YSO's, it is thought that this phenomenon may be one of the earliest (first $10^{5}$ years) identifiable stages of stellar evolution (of both massive and low-mass stars). The gas is not gravitationally bound, because for typical flow velocities of $10 \mathrm{~km} \mathrm{~s}^{-1}$ and sizes of a few tenths of a parsec, $10^{4} M_{\odot}$ is required to bind the gas; this is much more than the typical mass of a molecular cloud clump in which the flows occur. Other phenomena that are associated with molecular outflows are $\mathrm{HH}$-objects, $\mathrm{H}_{2} \mathrm{O}$ maser emission, and shock-excited $\mathrm{H}_{2}$ emission.

The flows are relatively cold $(10-20 \mathrm{~K})$, low-tomoderate density $\left(300-3000 \mathrm{~cm}^{-3}\right)$ streams of molecular gas with a mass in the range $0.01-200 M_{\odot}$, that are easily studied in the isotopomers of the $\mathrm{CO}$ molecule. From such observations it is found that clumping of material in the flow is common. Observations of molecules that trace higher-density regions $\left(10^{3}-10^{5} \mathrm{~cm}^{-3}\right)$ of a cloud (e.g. CS, HCN, SO) reveal (Tafalla et al. 1997) that higher densities do occur in the region of the flow, but that they are much more clumped than the CO. It is assumed that the molecular outflows are driven and directed by stellar jets (DeYoung 1986), rather than by winds originating from the star/disk system (Shu et al. 1991).

The kinetic energies that are associated with the flows are typically $10^{45} \mathrm{erg}$; the flow must therefore have a very important impact on the surrounding molecular cloud 
Table 1. Observed sources

\begin{tabular}{|c|c|c|c|c|c|c|c|c|c|}
\hline Source & $\begin{array}{c}\alpha(1950) \\
\mathrm{h} \mathrm{m} \mathrm{s}\end{array}$ & $\begin{array}{c}\delta(1950) \\
\circ, 11\end{array}$ & $\begin{array}{l}l \\
\circ\end{array}$ & $\begin{array}{l}b \\
\circ\end{array}$ & $\begin{array}{r}V_{\mathrm{lsr}} \\
\mathrm{km} \mathrm{s}^{-1} \\
\end{array}$ & $\begin{array}{l}\text { Dist. }^{1} \\
\text { kpc }\end{array}$ & $\begin{array}{l}L_{\text {fir }} \\
L_{\odot} \\
\end{array}$ & Ass. & Remarks \\
\hline WB89 1086 & 075951.4 & -281428 & 245.93 & 1.16 & 63.7 & 6.67 & 5400 & Bran96 & \\
\hline WB89 1099 & $0807 \quad 40.2$ & -355607 & 253.92 & -1.61 & 5.8 & 0.75 & 46 & $\begin{array}{l}\text { CG30-IRS4, HH120, } \\
\text { Bran111, DC253.3-1.6 }\end{array}$ & \\
\hline WB89 1135 & 082117.9 & -414610 & 259.61 & -2.70 & 54.6 & 6.42 & 14000 & & \\
\hline WB89 1173 & 083923.4 & -404118 & 260.78 & 0.68 & 7.4 & 1.35 & 480 & & \\
\hline WB89 1181 & 084449.4 & -434328 & 263.78 & -0.43 & 4.1 & 0.98 & 1630 & & \\
\hline WB89 1187 & 084701.3 & -432115 & 263.74 & 0.12 & 12.1 & 2.24 & 7100 & & \\
\hline WB89 1189 & 084739.4 & -430601 & 263.82 & 0.37 & 2.5 & 0.59 & 310 & & \\
\hline WB89 1262 & 091457.9 & -474350 & 270.26 & 0.84 & 9.5 & 3.40 & 38900 & $\begin{array}{l}\text { Bran246, RCW41, } \\
\text { G270.26+0.83 }\end{array}$ & only CS \\
\hline WB89 1275 & 092245.9 & -514636 & 274.01 & -1.15 & 36.9 & 6.38 & 936000 & Bran263, RCW42 & $\begin{array}{l}\text { only }{ }^{12} \mathrm{CO}, \\
{ }^{13} \mathrm{CO}\end{array}$ \\
\hline
\end{tabular}

${ }^{1}$ Kinematic distance, except for WB89 1086 and 1262 (photometric distance from Brand \& Blitz 1993).

medium. As a considerable amount of energy is being pumped into the clouds in this way, outflows are very important for our understanding of star formation as well as molecular cloud evolution.

The list of molecular outflow sources by Fukui et al. (1993) contains 164 sources; only 12 are in the southern hemisphere $\left(\delta<-20^{\circ}\right.$; excluding the $\rho$ Oph region). Observations of ${ }^{12} \mathrm{CO}$ towards IRAS sources have shown that in many cases there is a line wing that indicates the presence of outflows. During our survey in the outer Galaxy $\left(l: 85^{\circ}-280^{\circ}\right)$ of ${ }^{12} \mathrm{CO}$ towards IRAS sources with colours typical of star forming regions (Wouterloot \& Brand 1989 [WB89]), we have found about 150 sources $(14 \%)$ which in their spectra show broad features (wings) that are characteristic of outflow; including also the emission profiles which have a clear blue and/or red asymmetry, we find that $28 \%$ (about 300) of the detected sources have distinctly non-Gaussian line profiles. Mapping is required to confirm that broad $\mathrm{CO}$ profiles indeed represent outflow sources.

The Vela region $\left(245^{\circ}<l<275^{\circ}\right)$ contains a number of molecular cloud complexes with distances between several 100 pc and several kpc (see e.g. Murphy \& May 1991 and Liseau et al. 1992). In addition there are isolated clouds at larger distances, up to $15 \mathrm{kpc}$ (see Wouterloot et al. 1990). The WB89 survey in this region contains 208 IRAS sources, 41 of which were noted to have blue and/or red wings in their ${ }^{12} \mathrm{CO}(1-0)$ spectrum. From these, we selected eight sources for further study with the aim to derive size, mass, momentum, energy, dynamical timescale, and mechanical luminosity of the outflows in the sample, and to obtain size, temperature, and density of the molecular core from which they originate.

\section{Observations}

Between November 18-26, 1988 we used the 15-m SEST to observe ${ }^{12} \mathrm{CO}(1-0),{ }^{13} \mathrm{CO}(1-0), \mathrm{C}^{18} \mathrm{O}(1-0)$ and $\mathrm{CS}(2-1)$ towards seven sources. Towards one source we
Table 2. Observed transitions

\begin{tabular}{lrcc}
\hline Molecule & $\begin{array}{c}\text { Frequency } \\
(\mathrm{MHz})\end{array}$ & $\begin{array}{c}\mathrm{HPBW} \\
(\operatorname{arcsec})\end{array}$ & $\begin{array}{c}\mathrm{rms}^{1} \\
(\mathrm{~K})\end{array}$ \\
\hline${ }^{12} \mathrm{C}^{16} \mathrm{O}(1-0)$ & 115271.204 & 46 & $0.22(0.13)$ \\
${ }^{13} \mathrm{C}^{16} \mathrm{O}(1-0)$ & 110201.353 & 48 & $0.10(0.05)$ \\
${ }^{12} \mathrm{C}^{18} \mathrm{O}(1-0)$ & 109782.182 & 48 & $0.13(0.05)$ \\
${ }^{12} \mathrm{C}^{32} \mathrm{~S}(2-1)$ & 97980.968 & 54 & $0.20(0.10)$ \\
\hline
\end{tabular}

${ }^{1} \mathrm{rms}$ in brackets at IRAS PSC position (or outflow peak).

observed only ${ }^{12} \mathrm{CO}(1-0)$ and ${ }^{13} \mathrm{CO}(1-0)$ and towards one other source only $\mathrm{CS}(2-1)$ was observed.

We used a Schottky receiver in combination with a high-resolution AOS (channel width $43.3 \mathrm{kHz}$ ). We used frequency switching with an offset of 15 or $20 \mathrm{MHz}$, depending on the line width. Maps in ${ }^{12} \mathrm{CO}$ covering the outflow region were made on a raster with spacing of $20^{\prime \prime}$ near the IRAS source, and $40^{\prime \prime}$ in the outer parts of the map. Due to time constraints we have ${ }^{13} \mathrm{CO}$ data at only nine positions on a $40^{\prime \prime}$ raster for each source, centered on the IRAS PSC position. $\mathrm{C}^{18} \mathrm{O}$ and CS were traced on a $20^{\prime \prime}$ raster until the emission vanished in the noise. A loworder polynomial baseline was subtracted from the spectra before folding. The pointing was regularly checked on $\mathrm{SiO}$ masers; its rms uncertainty is $7^{\prime \prime}$. The observed sources are listed in Table 1. It gives the source name in Col. 1; the position in equatorial and galactic coordinates in Cols. 25 . The velocity of the main component is in Col. 6. In Cols. 7 and 8 are the kinematic distance (except for two sources for which a photometric distance is known) and bolometric luminosity (from WB89). Associated sources are shown in Col. 9. The observed transitions and frequencies are given in Cols. 1 and 2 of Table 2. The beam size of the SEST (Col. 3) is between $46^{\prime \prime}$ at $115 \mathrm{GHz}$ and $54^{\prime \prime}$ at $98 \mathrm{GHz}$. In Col. 4 we give the rms noise level in the spectra. All intensities in this paper are on the $T_{\mathrm{A}}^{*}$ scale. When deriving cloud masses and outflow parameters, we corrected for the main beam efficiency of the SEST ( 0.70 at all frequencies observed here). 

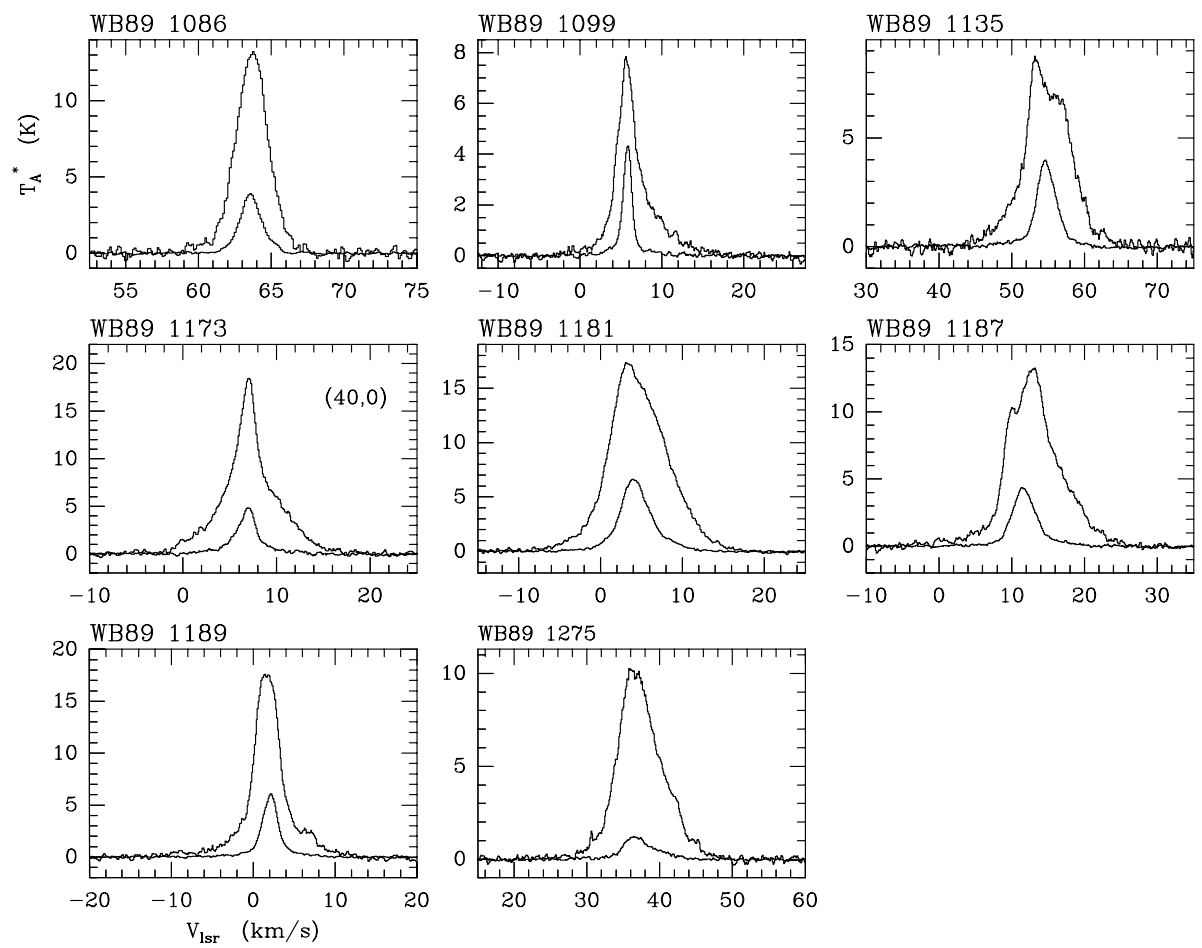

Fig. 1. ${ }^{12} \mathrm{CO}$ and ${ }^{13} \mathrm{CO}(1-0)$ spectra towards the positions with strong ${ }^{12} \mathrm{CO}$ line wing emission. The offset is $\left(0^{\prime \prime}, 0^{\prime \prime}\right)$, unless indicated otherwise

\section{Results}

\section{1. ${ }^{12} \mathrm{CO}$ and ${ }^{13} \mathrm{CO}$}

${ }^{12} \mathrm{CO}$ and ${ }^{13} \mathrm{CO}(1-0)$ spectra are shown in Fig. 1 (no line components have been subtracted; see below). The emission is shown on the same velocity scale for the eight sources towards the position with strong ${ }^{12} \mathrm{CO}$ line wing emission. For most sources this is at the IRAS PSC position (offset $\left(0^{\prime \prime}, 0^{\prime \prime}\right)$ ). An exception is WB89 1173 (offset $\left.\left(40^{\prime \prime}, 0^{\prime \prime}\right)\right)$. The ${ }^{12} \mathrm{CO}$ line profiles are much broader than those of ${ }^{13} \mathrm{CO}$, and extend over a large range in velocity (up to $25 \mathrm{~km} \mathrm{~s}^{-1}$ for WB89 1187), which is typical of outflow emission. In some of the ${ }^{13} \mathrm{CO}$ spectra wing emission is present as well. For three sources (WB89 1135, 1181, and 1187) the spectra also show a dip in the ${ }^{12} \mathrm{CO}$ emission at the velocity of the ${ }^{13} \mathrm{CO}$ peak, possibly a sign of self absorption, although for the latter source we conclude that the dip is caused by two partly overlapping velocity components (see further). A secondary maximum at $+7 \mathrm{~km} \mathrm{~s}^{-1}$ is visible in the spectrum towards WB89 1189, and is also due to another component, which is much stronger to the SE of the IRAS position.

In the maps of WB89 1099, 1135, 1173, 1187, 1189 and 1275 , we detected one or more other emission components (especially in ${ }^{12} \mathrm{CO}$ ), the velocity of which is often near that of the main component, hence causing confusion in identifying the outflowing gas. These components are identified at positions away from the center of the flows (where the main component of the emission tends to be narrower). Based on Gaussian fits made to the spectra at those positions, confusing components could also be subtracted from spectra taken towards the center of the outflows, where they generally almost disappear in the broad ${ }^{12} \mathrm{CO}$ profile. In some cases some weak residual emission may be left in the central, broad profiles, because the velocity of the confusing emission is too close to that of the main component, and an unambiguous Gaussian fit could not always be made.

The distribution of the velocity components that are unrelated to the outflows (and that were subtracted) is shown in Fig. 2. It is seen that these emission components do not have a maximum near the IRAS PSC positions, and for some sources they are only detected at the edge of the observed region. A few of those velocity components such as for WB89 $1099\left(4 \mathrm{~km} \mathrm{~s}^{-1}\right), 1187\left(10 \mathrm{~km} \mathrm{~s}^{-1}\right)$, and $1189\left(7 \mathrm{~km} \mathrm{~s}^{-1}\right)$ are rather extended.

To investigate whether cloud temperatures have a maximum near embedded heating (IRAS) sources, we have plotted in Fig. 3 the distribution of peak temperature for the sources mapped in ${ }^{12} \mathrm{CO}(1-0)$, after subtraction of unrelated (to the outflow) emission components. Towards six of the sources the maximum is within about $20^{\prime \prime}$ of the IRAS PSC position. Towards the two remaining sources the displacement of the peak of the temperature distribution appears to be significant (i.e. not due to noise) 

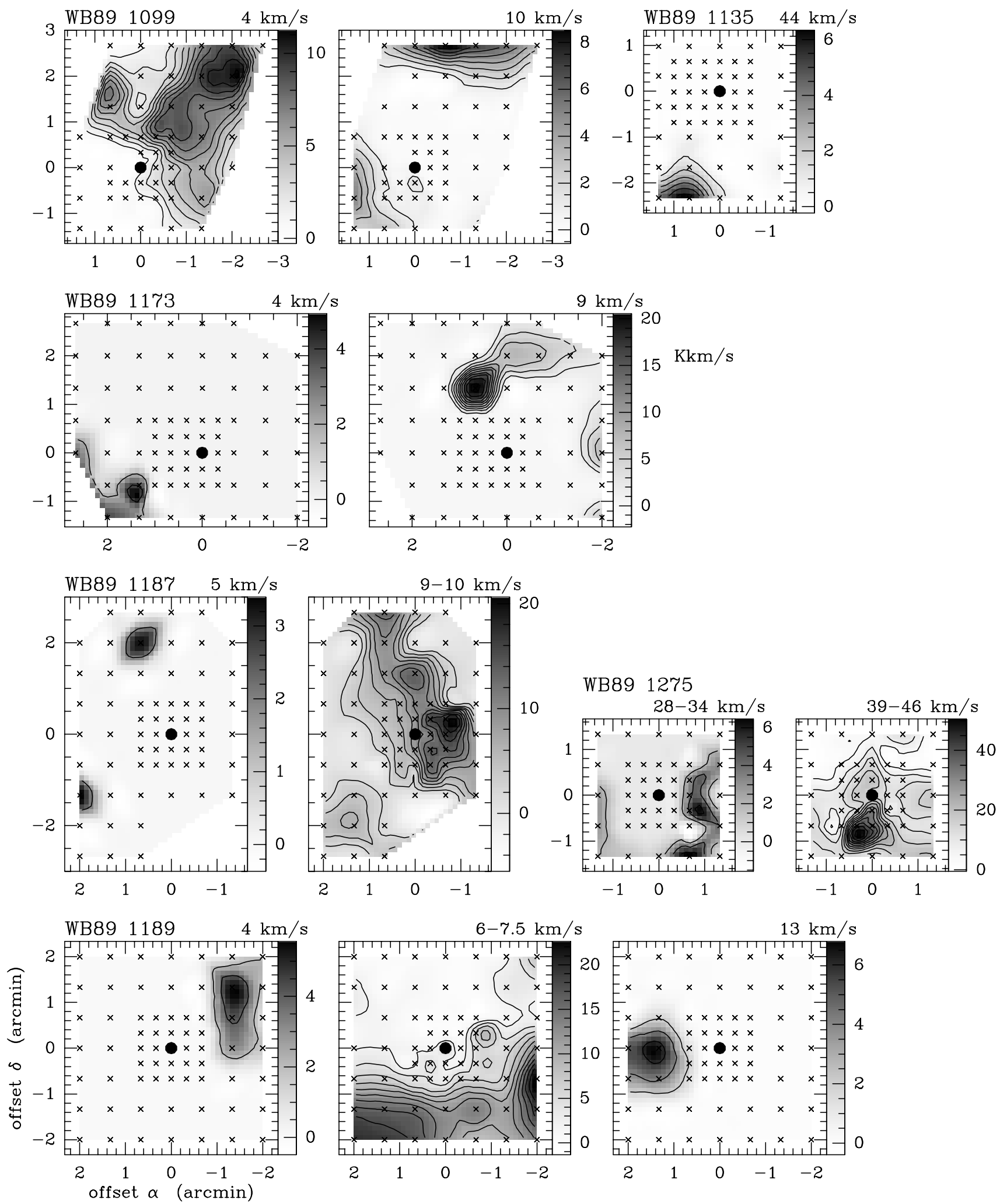

Fig. 2. The distribution of the ${ }^{12} \mathrm{CO}(1-0)$ emission of velocity components which are judged not to be associated with the outflows. The velocities of the unrelated components are indicated. Contour levels are 2 to $22 \mathrm{~K} \mathrm{~km} \mathrm{~s}^{-1}$ in steps of $2 \mathrm{~K} \mathrm{~km} \mathrm{~s}^{-1}$, except for WB89 1099 and 1135 where start value and contour interval are $1 \mathrm{~K} \mathrm{~km} \mathrm{~s}^{-1}$. Observed positions are indicated as crosses, the IRAS PSC position as filled circles 

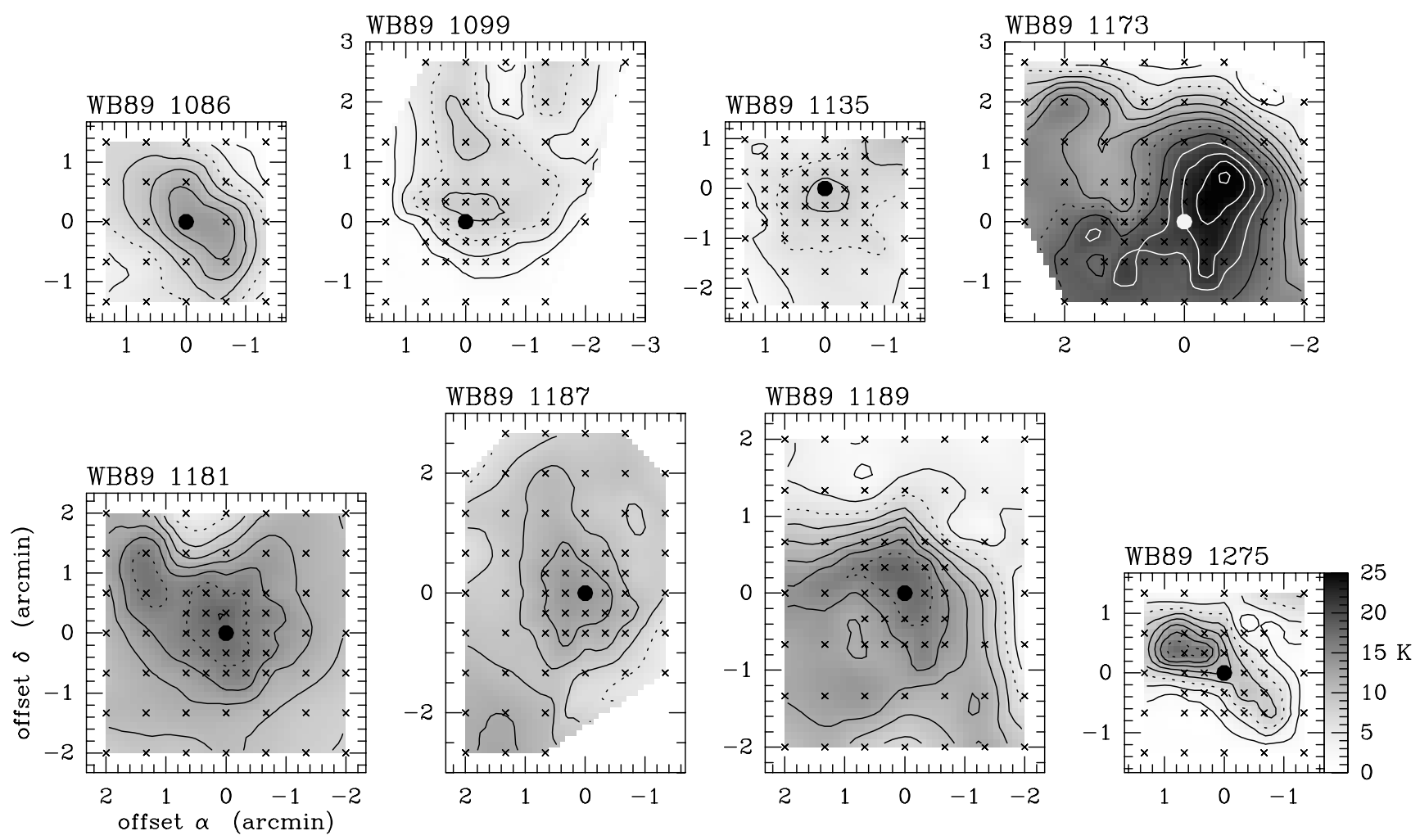

Fig. 3. The distribution of the ${ }^{12} \mathrm{CO}(1-0)$ peak $T_{\mathrm{A}}^{*}$ towards eight sources, after subtracting emission components that are unrelated to the outflow. Contour levels are 2 to $26 \mathrm{~K}$ in steps of $2 \mathrm{~K}$. Contours at 6 and $16 \mathrm{~K}$ are dotted and those of $20 \mathrm{~K}$ and higher are white. Observed positions are indicated as crosses, the IRAS PSC position as filled circles

compared to the beamsize, and amounts up to about $59^{\prime \prime}$ for WB89 1173 and 52" for WB89 1275 (resp. 0.14 and $1.6 \mathrm{pc})$. The distribution of large-scale CO emission associated with our sample of sources can be seen in the low resolution maps of Murphy \& May (1991), except for WB89 1086, 1135, and 1275, the velocities of which are outside the range discussed by these authors.

To study the outflowing gas, one integrates the "cleaned" (i.e. after subtracting the unrelated components) line profiles over some velocity interval, the outer limit $V_{\max }$ of which is taken for our sample of sources as the velocity where the emission first becomes less than $0 \mathrm{~K}$, and the inner limit $V_{\min }$ of which is taken at some distance from the line center in velocity space. The precise location of the inner velocity limit is determined by the shape and width of the line profile of the underlying quiescent gas. For the inner limit we have taken the velocity of the peak of the line profile, plus (for the red wing) or minus (blue wing) the half widths at half maximum intensity of the line profile: $\Delta v_{\text {blue }}$ or $\Delta v_{\text {red }}$. We took the smallest of both half widths, $\Delta v_{\mathrm{q}}=\min \left(\Delta v_{\text {blue }}, \Delta v_{\text {red }}\right)$, and to correct the wing emission for the contribution of the quiescent gas, we subtracted at each channel between $V_{\min }$ and $V_{\max }$ the contribution of a Gaussian with a width equal to $2 \times \Delta v_{\mathrm{q}}$, and a velocity equal to the velocity of the peak temperature of the line profile. The resulting distribution of the red and blue wing emission is shown in Fig. 4.

Except for WB89 1173 (blue wing), the outflow emission (i.e. ${ }^{12} \mathrm{CO}$ ) is centered near (i.e. within one beam of) the IRAS $(0,0)$ position, and moderately extended compared to the $46^{\prime \prime} 115 \mathrm{GHz}$ beam. For some sources the wing emission is very small (and possibly nonexistent), such as the blue and red wing of WB89 1086, and the blue wings of WB89 1099 and WB89 1189 (although for the latter source there is clearly some weak wing emission present in Fig. 1). For the other sources with substantial wing emission one can distinguish sources where the distribution of blue and red wing emission is similar, such as WB89 1173, and sources where the emission is bipolar (e.g. WB89 1187, where the blue (red) wing has a maximum east (west) of the IRAS PSC position). Also for WB89 1135 there appears such a difference in the location of the wing emission. Towards WB89 1181 the red wing is well defined with a peak east of the PSC position, but the situation for the blue wing is less clear.

We have derived parameters of the outflows, following Snell et al. (1984), and assuming $T_{\mathrm{ex}}=20 \mathrm{~K}, \tau=1$, and 

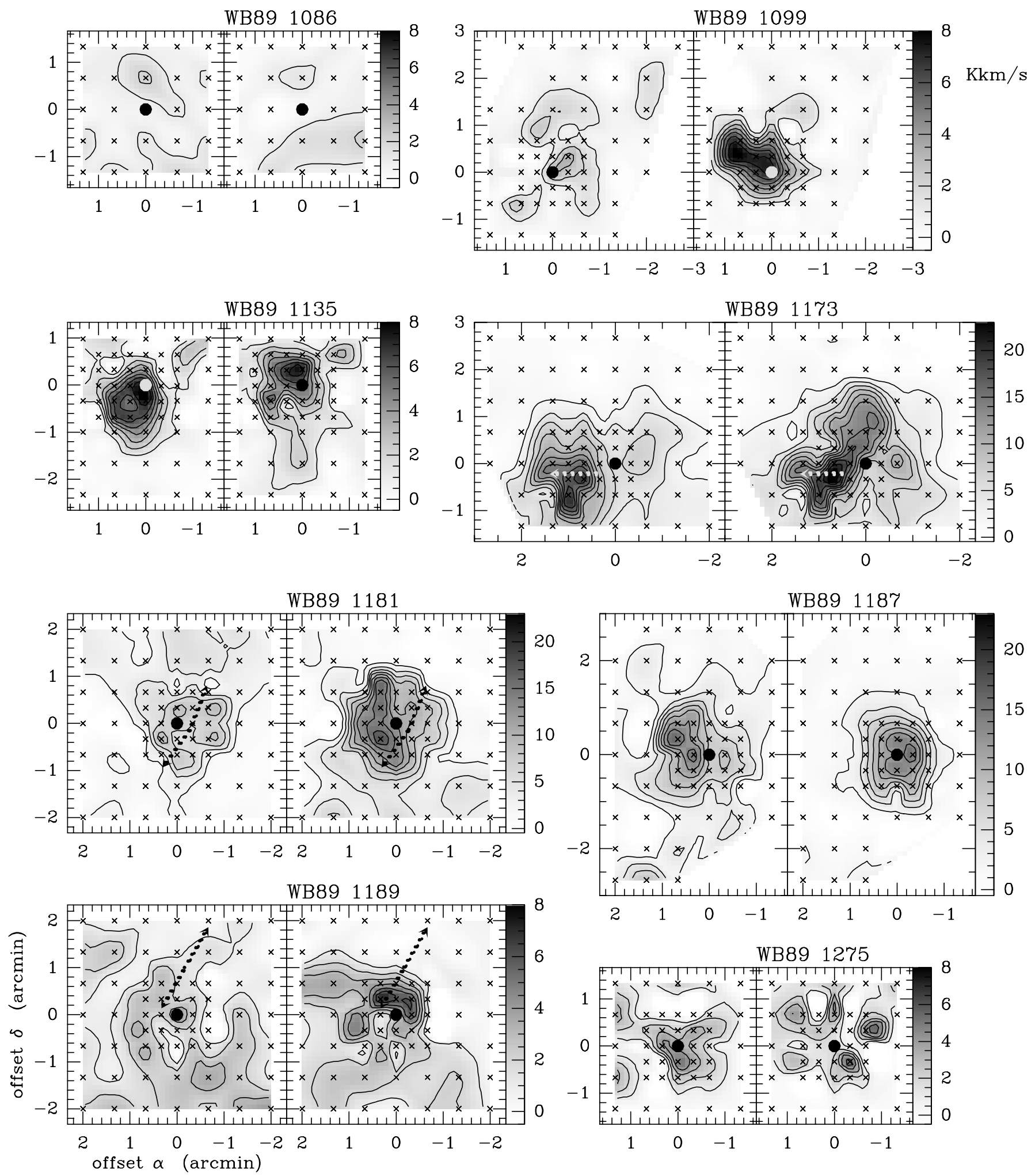

Fig. 4. The distribution of the integrated blue (left) and red (right) ${ }^{12} \mathrm{CO}(1-0)$ line wing emission towards eight sources. The lowest contour level and the contour interval are $1 \mathrm{~K} \mathrm{~km} \mathrm{~s}^{-1}$, except for WB89 1173, 1181, and 1187 where they are $2 \mathrm{~K} \mathrm{~km} \mathrm{~s}{ }^{-1}$. Observed positions are indicated as crosses, the IRAS PSC position as filled circles. The arrows indicate for WB89 1173, 1181, and 1189 the direction of $\mathrm{H}_{2}$ jets detected by Massi et al. (1997) 
LTE (see Wouterloot et al. 1989; Shepherd \& Churchwell 1996). The results are listed in Table 3. Column 1 gives the source name. Column 3 the largest velocity range of the wing emission, reached at the position listed in Col. 4. We derived the velocity range from the velocity at which the emission first reaches the $0 \mathrm{~K}$ level, and distinguish between the blue and red wings and the total spectrum. The blue and red wings can show their largest range at different positions and therefore the position with the largest total velocity range can also differ from that of the largest blue or red range. In Col. 5 is the median value of the line width of the subtracted line component of the quiescent gas. The standard deviation of this line width distribution at all positions in the maps is 0.5 to $1 \mathrm{~km} \mathrm{~s}^{-1}$, and in some sources this width increases slightly with peak $T_{\mathrm{A}}^{*}$, probably due to contributions of the outflow which cannot be separated from the quiescent gas. Column 6 lists the offsets with strongest blue and red wing emission (largest $\int T_{\mathrm{A}}^{*} \mathrm{~d} v$ ). In general the maximum outflow velocities (Col. 3 of Table 3 ) do not occur at positions where the total wing area (Col. 6) is largest. Probably this is due to the fact that only small part of the outflow is at the highest velocities, and due to projection effects. In Cols. 7 to 12 are the derived outflow mass, momentum and energy (for blue and red wing and the total emission), the diameter (corrected for beamsize), average outflow velocity and the dynamical timescale of the outflow. The ratio of energy and timescale is the mechanical luminosity in Col. 13.

From the maps and the spectra we concluded that there is no outflow emission towards WB89 1086. This is a source showing only one line component which is slightly broader at several positions and showing only a small range in velocity with emission (see Col. 3 of Table 3 ). Towards WB89 1275 we derive only parameters for the blue wings because of confusion due to emission at higher velocities $\left(39-46 \mathrm{~km} \mathrm{~s}^{-1}\right.$ - see Fig. 2) which may not have been correctly subtracted.

The outflow masses range from $1 M_{\odot}$ for WB89 1099 to $150 M_{\odot}$ for WB89 1135, the sources with the lowest and highest FIR luminosity in the sample which were mapped in CO (apart from WB89 1275, for which $L_{\mathrm{FIR}}$ is higher, but for which we could only study the blue wing emission). Also the outflow momentum and energy show extreme values towards these sources. The outflow mass in WB89 1135 is among the highest ones found anywhere (about $200 M_{\odot}$; see e.g. Bachiller 1996). From the masses and diameters of the outflows we obtain $\mathrm{H}_{2}$ densities ranging from $120 \mathrm{~cm}^{-3}$ (red wing of WB89 1135) to $1590 \mathrm{~cm}^{-3}$ (red wing of WB89 1099). The median value is $850 \mathrm{~cm}^{-3}$, which is typical for such sources (Lada 1985). The dynamical timescale in Col. 12 ranges from about $0.510^{5}$ years to $410^{5}$ years, which is at the upper range of values found in such sources (see e.g. Fig. 12 in Shepherd \& Churchwell 1996). A discussion of the accuracy of outflow parameters and a comparison of different methods to derive these pa- rameters is given by Cabrit \& Bertout (1990). The outflow velocity in Col. 11 of Table 3 is a average value, weighted with $T_{\mathrm{A}}^{*}$ at all positions. The maximum outflow velocity in Col. 3 is larger by a factor 3.8 (median; range from 3.0 [red wing in WB89 1099] to 6.6 [blue wing in WB89 1099]). Using this velocity would result in a dynamical timescale smaller- and a mechanical luminosity larger by this factor.

\section{2. $C S$ and $C^{18} \mathrm{O}$}

Towards all sources which we observed in those molecules, we detected $\mathrm{C}^{18} \mathrm{O}(1-0)$ and $\mathrm{CS}(2-1)$ emission. Spectra at peak positions are shown in Fig. 5. For some sources the peak positions are displaced from the positions with strongest outflow emission or from the IRAS PSC position. In most cases $\mathrm{C}^{18} \mathrm{O}$ is weaker than $\mathrm{CS}$. The exception is WB89 1086 where both lines have approximately equal intensity and both are faint. We note that the CS and/or $\mathrm{C}^{18} \mathrm{O}$ linewidths for four of the sources (WB89 1086, 1099, 1173 , and 1189$)$ are smaller $\left(1.3 \mathrm{~km} \mathrm{~s}^{-1}\right)$ than for the others $\left(2.6 \mathrm{~km} \mathrm{~s}^{-1}\right)$. The same is seen in the values of $\Delta v_{\mathrm{q}}$ of the quiescent ${ }^{12} \mathrm{CO}$ gas (Col. 5 of Table 3 ). This might be related to the smaller FIR luminosity of the IRAS sources $\left(46-5400 L_{\odot}\right.$ vs. $\left.1630-936000 L_{\odot}\right)$, although these luminosity ranges show some overlap.

The results from the Gaussian fits are summarized in Table 4, where we list the values for $\mathrm{CS}$ and $\mathrm{C}^{18} \mathrm{O}$ for the average cloud spectrum and towards the peak position. Six of the sources in Table 1 were observed in $\operatorname{CS}(2-1)$ by Bronfman et al. (1996) at the IRAS PSC position. Those not observed are WB89 1086, WB89 1173, and WB89 1187. We did not observe CS (nor $\mathrm{C}^{18} \mathrm{O}$ ) towards WB89 1275. Regarding the latter source, the weak line $\left(T_{\mathrm{mb}}=0.4 \mathrm{~K}\right)$ detected by Bronfman et al. (compared to the peak CS temperatures in Table 4) might be caused by a clump of which the center is displaced from the PSC position, as is suggested by Fig. 3 .

The $\mathrm{CS}(2-1)$ and $\mathrm{C}^{18} \mathrm{O}(1-0)$ spectra do not show other velocity components. However, outflow emission is visible in some of the CS(2-1) spectra, e.g. WB89 1099 (see also Nielsen et al. 1998), 1135 and 1181. Also towards WB89 1262, not observed in CO, CS shows line wings. However the signal-to-noise ratio is not good enough at all positions to distinguish between the distributions of outflow and quiescent gas in those four objects. We made Gaussian fits to the $\mathrm{CS}(2-1)$ and $\mathrm{C}^{18} \mathrm{O}(1-0)$ spectra, and the resulting $\int T_{\mathrm{A}}^{*} \mathrm{~d} v$ distributions are shown Fig. 6. Those of the peak $T_{\mathrm{A}}^{*}$ are essentially identical in most cases. Sources where the distribution of peak $T_{\mathrm{A}}^{*}$ is different are shown in Fig. 7. In most sources the two molecules show a maximum at or close to the PSC position. An exception is the CS emission from WB89 1086, which is rather weak towards $(0,0)$ and shows somewhat stronger emission northeast of the PSC position. WB89 1173 shows a maximum of $\int T_{\mathrm{A}}^{*} \mathrm{~d} v$ of CS at $(0,0)$, but the maximum of the distribution of $T_{\mathrm{A}}^{*}$ is displaced to the offset $\left(37^{\prime \prime},-35^{\prime \prime}\right)$. In 
Table 3. Outflow parameters derived from ${ }^{12} \mathrm{CO}(1-0)$ observations

\begin{tabular}{|c|c|c|c|c|c|c|c|c|c|c|c|c|}
\hline $\begin{array}{c}\text { Source } \\
\text { WB89 } \\
(1) \\
\end{array}$ & $\begin{array}{l}\text { Wing } \\
(2) \\
\end{array}$ & $\begin{array}{r}V_{\text {range }} \\
\mathrm{km} \mathrm{s}^{-1} \\
(3) \\
\end{array}$ & $\begin{array}{c}\text { offset } \\
\text { " } \\
(4) \\
\end{array}$ & $\begin{array}{r}\Delta v_{\mathrm{q}} \\
\mathrm{km} \mathrm{s}^{-1} \\
(5) \\
\end{array}$ & $\begin{array}{c}\int T_{\mathrm{A}}^{*} \mathrm{~d} v \\
\text { peak }\left({ }^{\prime \prime}\right) \\
(6)\end{array}$ & $\begin{array}{r}\text { Mass } \\
M_{\odot} \\
(7) \\
\end{array}$ & $\begin{array}{r}\text { Mom. } \\
M_{\odot} \mathrm{km} \mathrm{s}^{-1} \\
(8) \\
\end{array}$ & $\begin{array}{r}\text { Energy } \\
10^{44} \text { erg } \\
(9) \\
\end{array}$ & $\begin{array}{r}\text { Diam. } \\
\mathrm{pc} \\
(10) \\
\end{array}$ & $\begin{array}{r}V_{\text {out }} \\
\mathrm{km} \mathrm{s}^{-1} \\
(11) \\
\end{array}$ & $\begin{array}{r}t_{\mathrm{dyn}} \\
10^{5} \mathrm{yr} \\
(12) \\
\end{array}$ & $\begin{array}{r}L_{\text {mech }} \\
L_{\odot} \\
(13) \\
\end{array}$ \\
\hline \multirow{3}{*}{1086} & blue & 5.1 & $(40,40)$ & & & - & - & - & - & - & - & \\
\hline & red & 5.3 & $(-40,0)$ & & & - & - & - & - & - & - & \\
\hline & total & 9.4 & $(-40,0)$ & 1.80 & & - & - & - & - & - & - & - \\
\hline \multirow[t]{3}{*}{1099} & blue & 10.4 & $(0,0),(40,20)$ & & $(-22,16)$ & 0.48 & 0.71 & 0.15 & 0.37 & 1.57 & 1.15 & \\
\hline & red & 7.3 & $(0,0)$ & & $(46,22)$ & 0.59 & 1.26 & 0.45 & 0.22 & 2.46 & 0.44 & \\
\hline & total & 17.7 & $(0,0)$ & 1.13 & $(-3,3)$ & 1.07 & 1.97 & 0.60 & & & 0.65 & 0.0076 \\
\hline \multirow[t]{3}{*}{1135} & blue & 10.9 & $(0,0)$ & & $(6,-10)$ & 74 & 226 & 93 & 2.44 & 3.51 & 3.42 & \\
\hline & red & 11 & $(20,-20)$ & & $(6,20)$ & 78 & 193 & 68 & 2.65 & 3.10 & 4.22 & \\
\hline & total & 21.5 & $(40,0)$ & 2.71 & $(6,2)$ & 151 & 419 & 161 & & & 3.79 & 0.36 \\
\hline \multirow[t]{3}{*}{1173} & blue & 10.8 & $(60,0)$ & & $(58,-46)$ & 13 & 30 & 9.5 & 0.76 & 2.53 & 1.47 & \\
\hline & red & 10.5 & $(60,-20)$ & & $(38,-16)$ & 15 & 28 & 8.2 & 0.75 & 2.18 & 1.70 & \\
\hline & total & 19.3 & $(60,-20)$ & 2.03 & $(45,-15)$ & 28 & 58 & 18 & & & 1.58 & 0.094 \\
\hline \multirow[t]{3}{*}{1181} & blue & 13.4 & $(0,0)$ & & $(19,-19)$ & 6.8 & 23 & 9.5 & 0.59 & 3.85 & 0.76 & \\
\hline & red & 12.5 & $(0,0)$ & & $(19,-19)$ & 7.7 & 26 & 11 & 0.55 & 3.96 & 0.68 & \\
\hline & total & 25.9 & $(0,0)$ & 3.16 & $(18,-18)$ & 14 & 49 & 20 & & & 0.72 & 0.44 \\
\hline \multirow[t]{3}{*}{1187} & blue & 13.1 & $(0,0)$ & & $(44,19)$ & 29 & 82 & 38 & 1.23 & 3.26 & 1.87 & \\
\hline & red & 15.6 & $(40,40)$ & & $(-16,0)$ & 26 & 89 & 39 & 1.16 & 4.14 & 1.88 & \\
\hline & total & 25.3 & $(0,0)$ & 2.82 & $(20,0)$ & 55 & 171 & 77 & & & 1.60 & 0.40 \\
\hline \multirow[t]{3}{*}{1189} & blue & 8.3 & $(20,20)$ & & $(0,0)$ & 1.2 & 2.0 & 0.47 & 0.32 & 2.19 & 0.72 & \\
\hline & red & 7.5 & $(-40,20)$ & & $(19,25)$ & 1.2 & 1.8 & 0.36 & 0.31 & 1.69 & 0.90 & \\
\hline & total & 15.4 & $(20,20)$ & 1.80 & $(18,25)$ & 2.3 & 3.7 & 0.83 & & & 0.80 & 0.0086 \\
\hline \multirow[t]{3}{*}{1275} & blue & 12.3 & $(40,40)$ & & $(-6,-18)$ & 66 & 255 & 119 & 2.13 & 3.77 & 2.78 & 0.36 \\
\hline & red & 10.5 & $(20,40)$ & & & 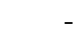 & - & - & - & - & - & \\
\hline & total & 21.5 & $(40,40)$ & 3.61 & & - & - & - & - & - & - & - \\
\hline
\end{tabular}
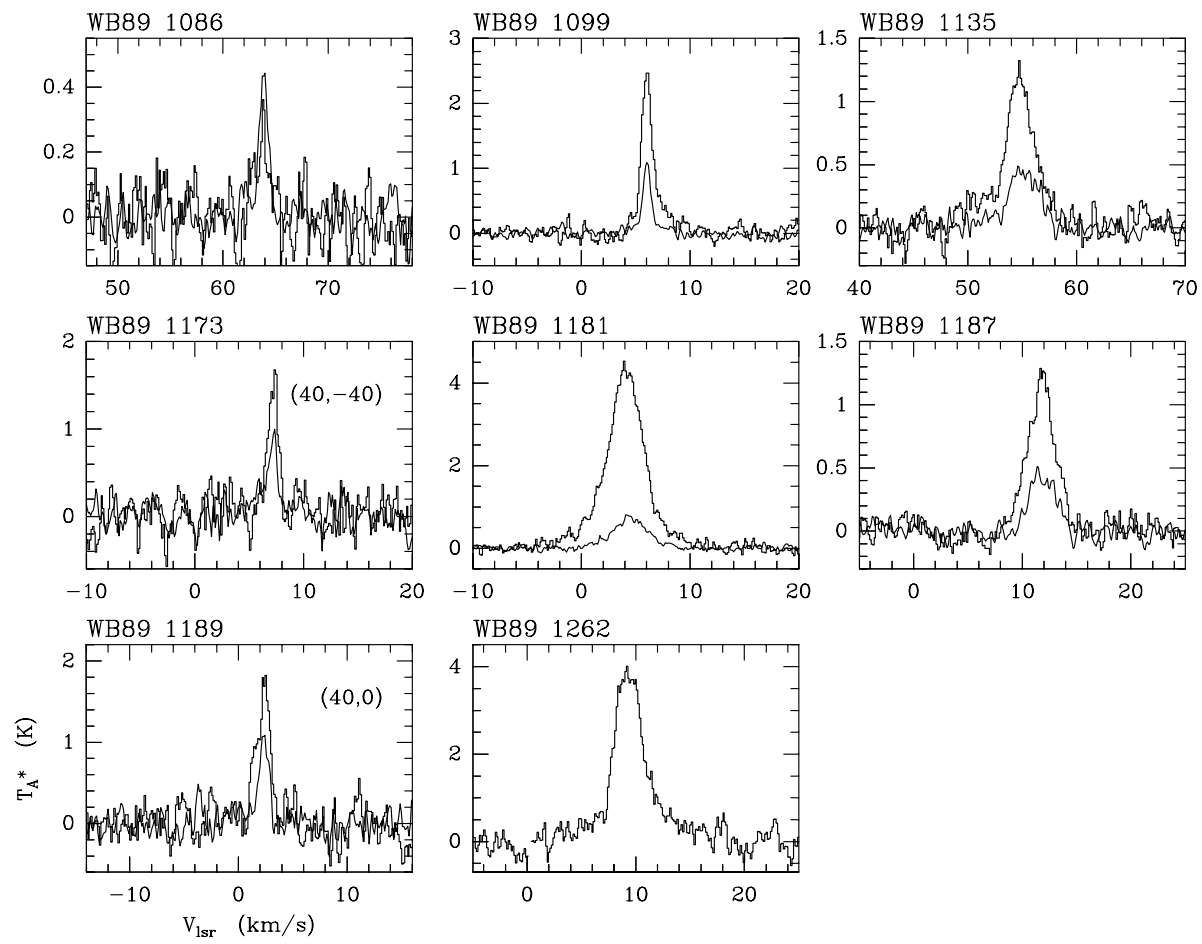

5 WB89 1187
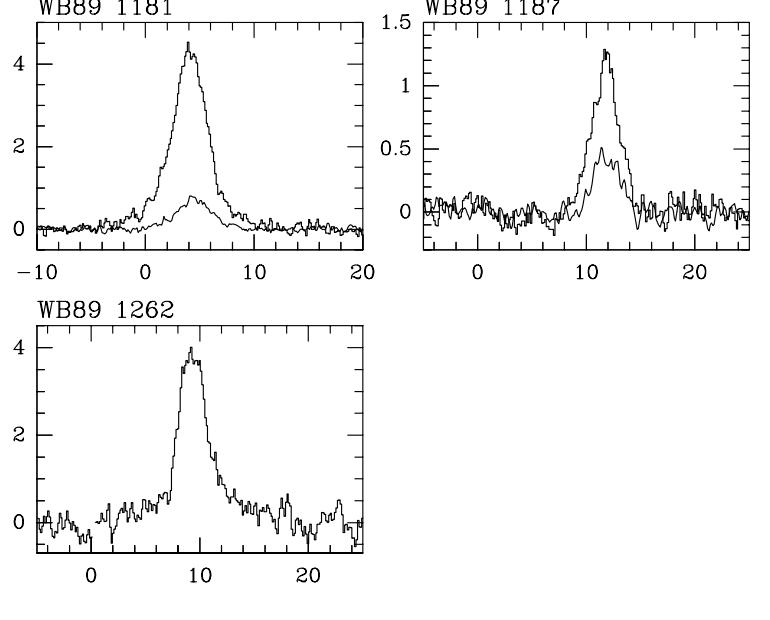

Fig. 5. $\mathrm{C}^{18} \mathrm{O}(1-0)$ and $\mathrm{CS}(2-1)$ spectra towards positions with maximum intensity. CS is plotted as a histogram and is the strongest line for all sources. The offset is $\left(0^{\prime \prime}, 0^{\prime \prime}\right)$, unless indicated otherwise 

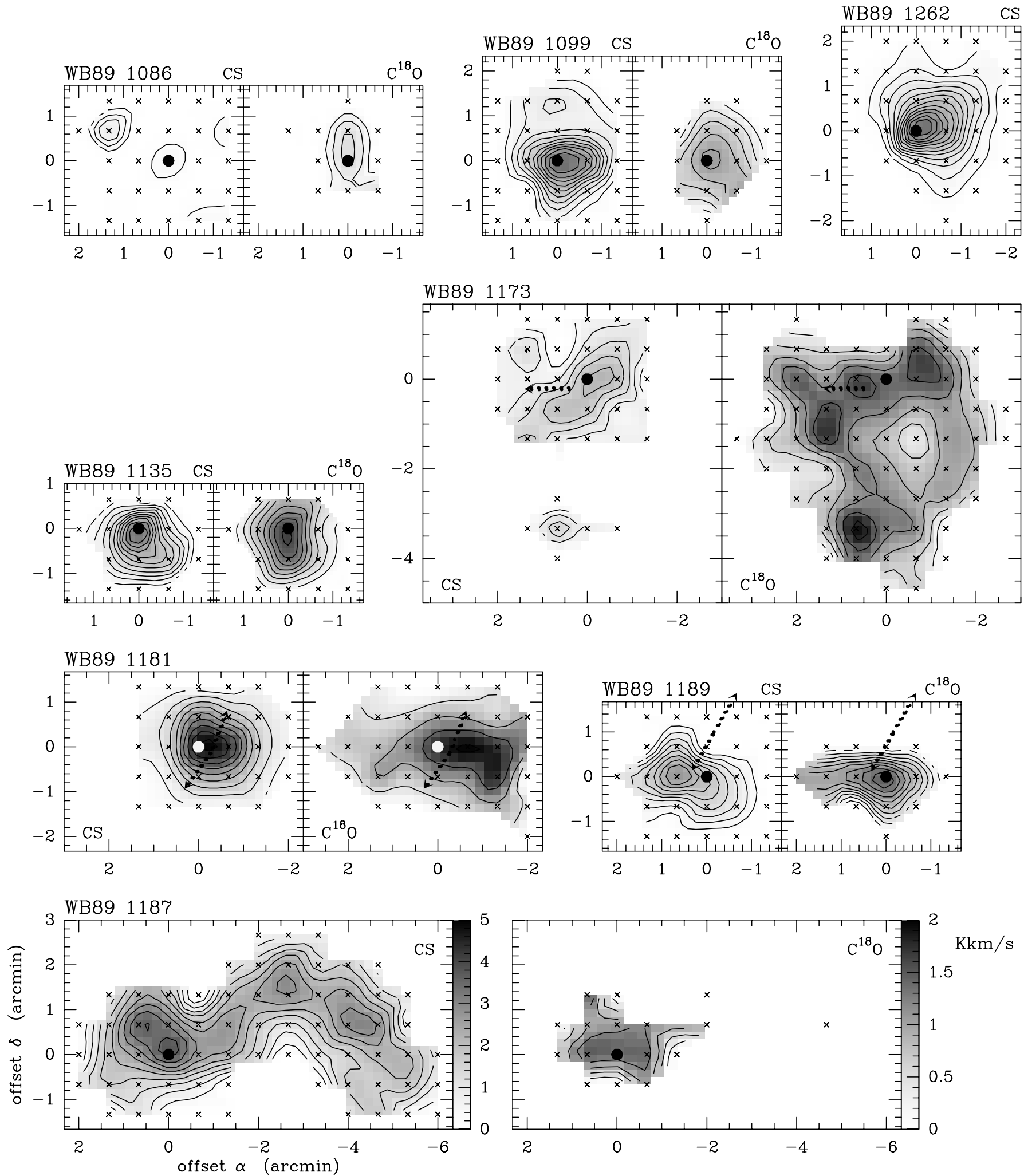

Fig. 6. The distribution of $\int T_{A}^{*} \mathrm{~d} v$ of $\mathrm{CS}(2-1)$ and $\mathrm{C}^{18} \mathrm{O}(1-0)$. The observed positions are indicated by crosses, and the IRAS PSC position by a filled circle. Lowest contour level and contour step are for CS and $\mathrm{C}^{18} \mathrm{O}: 0.2$ and $0.15 \mathrm{~K} \mathrm{~km} \mathrm{~s}^{-1}$ (WB89 1086); 0.3 and $0.2 \mathrm{~K} \mathrm{~km} \mathrm{~s}^{-1}$ (WB89 1099); 0.3 and $0.2 \mathrm{~K} \mathrm{~km} \mathrm{~s}^{-1}$ (WB89 1135); 0.4 and $0.3 \mathrm{~K} \mathrm{~km} \mathrm{~s}^{-1}$ (WB89 1173); 2 and $0.8 \mathrm{Kkm} \mathrm{s}{ }^{-1}$ (WB89 1181); 0.4 and $0.3 \mathrm{~K} \mathrm{~km} \mathrm{~s}^{-1}$ (WB89 1187); 0.3 and $0.15 \mathrm{~K} \mathrm{~km} \mathrm{~s}^{-1}$ (WB89 1189); $1 \mathrm{~K} \mathrm{~km} \mathrm{~s}^{-1}$ (WB89 1262). The greyscale range is $0-5 \mathrm{~K} \mathrm{~km} \mathrm{~s}^{-1}(\mathrm{CS})$ or $0-2 \mathrm{~K} \mathrm{~km} \mathrm{~s}^{-1}\left(\mathrm{C}^{18} \mathrm{O}\right)$, except for WB89 1181 and 1262 , where it is $0-19 \mathrm{~K} \mathrm{~km} \mathrm{~s}^{-1}$ (CS) and $0-3.3 \mathrm{~K} \mathrm{~km} \mathrm{~s}^{-1}\left(\mathrm{C}^{18} \mathrm{O}\right)$. The arrows indicate for WB89 1173, 1181, and 1189 the direction of $\mathrm{H}_{2}$ jets detected by Massi et al. $(1997)$ 

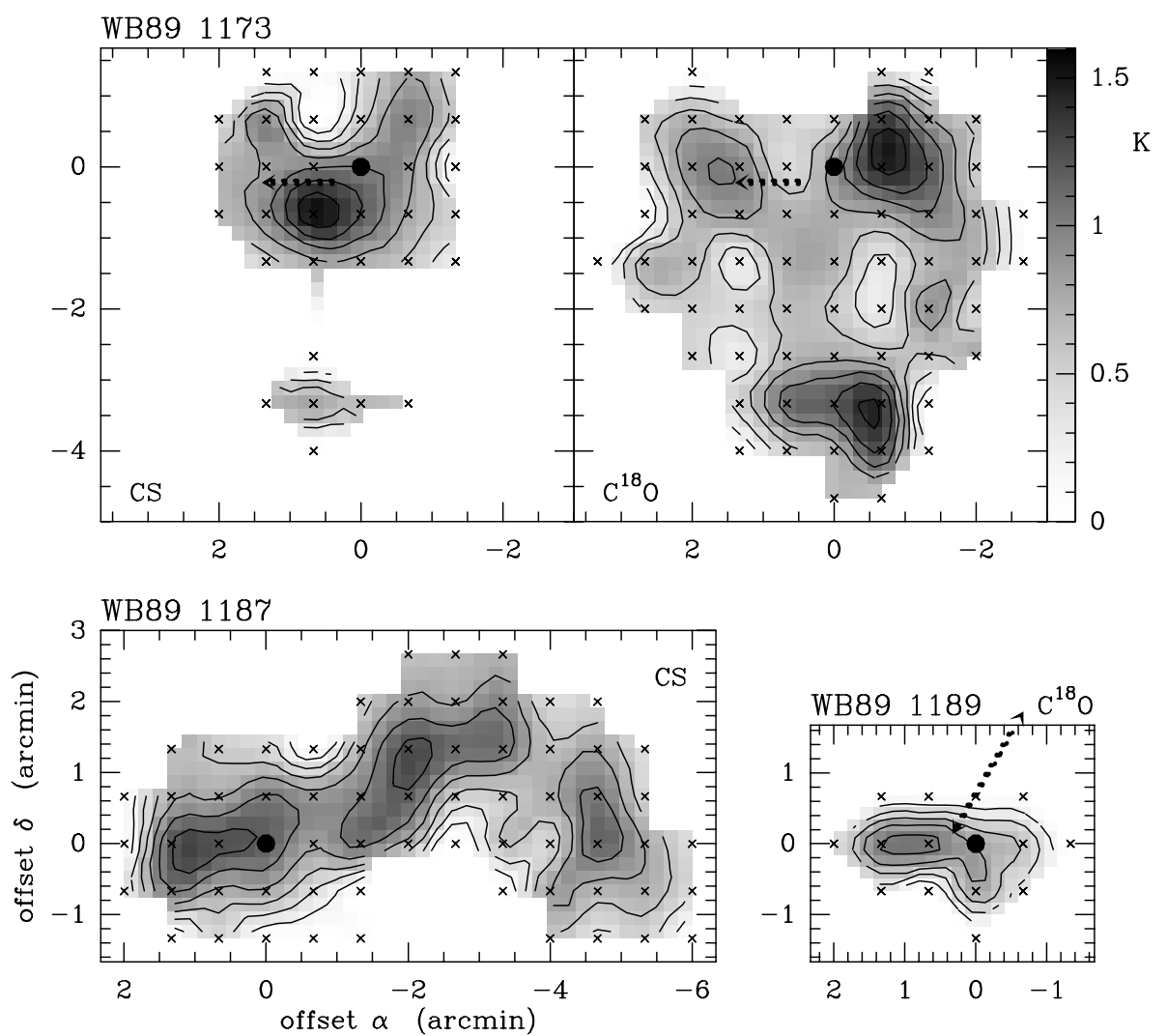

Fig. 7. The distribution of peak $T_{\mathrm{A}}^{*} \mathrm{~d} v$ of $\mathrm{CS}(2-1)$ and $\mathrm{C}^{18} \mathrm{O}(1-0)$ for some of the sources. The observed positions are indicated by crosses, and the IRAS PSC position by a filled circle. Lowest contour level and contour step are both for CS and $\mathrm{C}^{18} \mathrm{O} 0.2 \mathrm{~K}$. The arrows indicate for WB89 1173 and 1189 the direction of $\mathrm{H}_{2}$ jets detected by Massi et al. (1997)

$\mathrm{C}^{18} \mathrm{O}$ this source shows a number of peaks displaced from the PSC position. The maximum in WB89 1181 is slightly to the west of the PSC position, the CS emission peaks closer to $(0,0)$ than the $\mathrm{C}^{18} \mathrm{O}$, which is at $\left(-41^{\prime \prime}, 0^{\prime \prime}\right)$ (peak $\left.T_{\mathrm{A}}^{*}\right)$ or $\left(-60^{\prime \prime},-24^{\prime \prime}\right)\left(\int T_{\mathrm{A}}^{*} \mathrm{~d} v\right)$. The CS distribution near WB89 1187 shows a chain of three clumps, the most eastern one of which coincides with the IRAS PSC position; The strongest $T_{\mathrm{A}}^{*}$ is towards the eastern one at $\left(65^{\prime \prime},-6^{\prime \prime}\right)$. Offsets mentioned above can differ from those in Table 4 because they were derived from the contour maps rather than from the individual spectra.

Towards four of the sources, Massi et al. (1997) searched for and detected NIR $\mathrm{H}_{2}$ emission: WB89 1173, 1181, 1187, and 1189. We have indicated for three of those sources (using updated information from Massi 1999) their direction as $1^{\prime}$ arrows in Figs. 4, 6, and 7. The origin (for WB89 1173) or the centers of the arrows coincide with the embedded (NIR) objects proposed by Massi et al. (1997) to be the jets' origin. It seems that in all cases the direction is approximately parallel to the short axis of the $\mathrm{CS}$ or $\mathrm{C}^{18} \mathrm{O}$ clumps. In none of the three cases the direction is that of one of the outflows found in $\mathrm{CO}$ (see Fig. 4). These $\mathrm{H}_{2}$ features are extremely small (some arcseconds) for WB89 1173 and 1189, and for the latter source this emission is outside the $\mathrm{CS}$ and $\mathrm{C}^{18} \mathrm{O}$ clumps (see Fig. 6). On the other hand, in WB89 1181 nine individual $\mathrm{H}_{2}$ features can be distinguished, up to $30^{\prime \prime}$ northwest and southeast from an embedded star, which is close to the IRAS PSC position and lies inside the dense clumps. The $\mathrm{H}_{2}$ emission towards WB89 1187 is very weak.

We can compare the locations of $\mathrm{CS}$ and $\mathrm{C}^{18} \mathrm{O}$ clumps in Fig. 6 with the distribution of ${ }^{12} \mathrm{CO}(1-0)$ line wings in Fig. 4. Although in both figures the structures seen are not much larger than the angular resolution of the observations, there are a few sources where the cloud cores are clearly separated from the outflows (projected on the sky). Examples are the red wing of WB89 1099, both wings of WB89 1181, and the red wing of WB89 1187, where the outflow gas thus moves away from the dense cores. The situation is less clear towards the other sources, possibly due to projection effects.

From our data we derived the clump parameters listed in Table 5. The radii in Cols. $2(\mathrm{CS})$ and $4\left(\mathrm{C}^{18} \mathrm{O}\right)$ were obtained from the area in the grid where these molecules were detected: $r=\sqrt{\text { Area } / \pi}$. This radius was corrected for the beam size. There was not always enough time to observe a large enough area of sky, hence for some clumps the values are lower limits. This applies mostly to CS in WB89 1173 and to $\mathrm{C}^{18} \mathrm{O}$ in WB89 1187 (but it is possible 

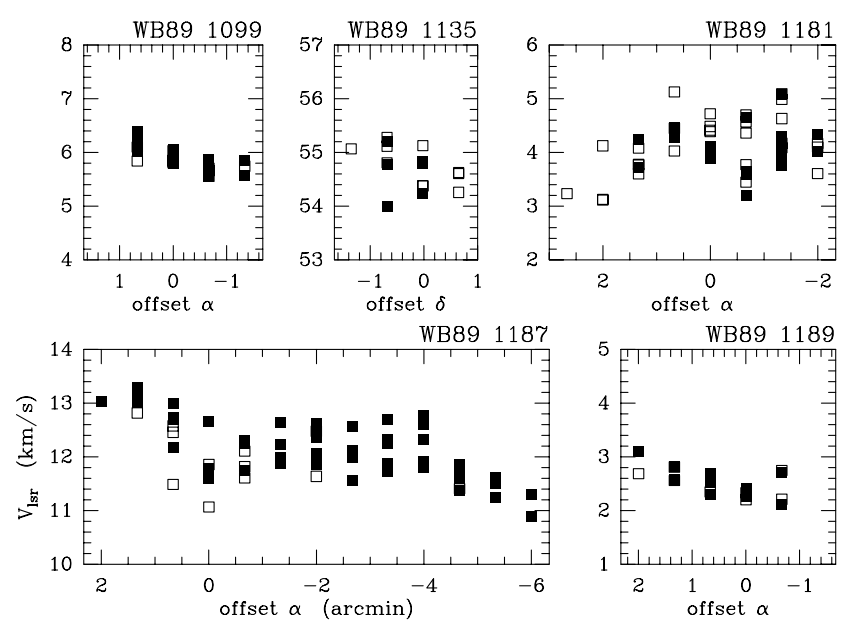

Fig. 8. Position $(\Delta \alpha$ or $\Delta \delta)$ - velocity diagram for five clouds with results from Gaussian fits. Filled symbols indicate CS data; open symbols $\mathrm{C}^{18} \mathrm{O}$ data

that near the other sources there are also more clumps which we did not trace). We applied another method to derive the clump radii in Cols. 3 and 5. Here we used their area at the half-intensity level. These values are smaller of course than those in Cols. 2 and 4. Because clouds are not spherical in most cases, all numbers should be considered as equivalent cloud radii. The virial mass in Cols. 6 and 7 was derived assuming a density distribution proportional to $r^{-2}\left(M_{\mathrm{vir}}=126 r \Delta v^{2}\right.$; see e.g. MacLaren et al. 1988), and here we used the radii in Cols. 2 and 4. The values derived from $\mathrm{C}^{18} \mathrm{O}$ can be compared with the LTE masses in Col. 8, which were derived assuming $\mathrm{C}^{18} \mathrm{O}$ is optically thin (using $N\left(\mathrm{H}_{2}\right)=3.010^{16} T_{\mathrm{ex}} \int T_{\mathrm{A}}^{*}\left(\mathrm{C}^{18} \mathrm{O}\right) / 0.7 \mathrm{~d} v$; Wilson et al. 1986). We used a ratio ${ }^{12} \mathrm{CO} / \mathrm{C}^{18} \mathrm{O}$ of 560 (Wilson \& Rood 1994), an excitation temperature $T_{\text {ex }}$ of $20 \mathrm{~K}$, a ${ }^{12} \mathrm{CO} / \mathrm{H}_{2}$ abundance of $10^{-4}$, and multiplied by a factor 1.36 to take He into account (0.7 is the SEST main beam efficiency). For most clouds the $M_{\text {vir }}$ is the larger mass. The differences are not larger than typically found for such objects, however. If one uses the conversion factor from $N\left(\mathrm{C}^{18} \mathrm{O}\right)$ to $N\left(\mathrm{H}_{2}\right)$ found by Frerking et al. (1982), instead of the above mentioned abundance ratios, the masses would become about a factor 2 larger. From the masses in Col. 8 and the cloud radii in Col. 4 we obtain the average density in Col. 9. If we had used the radii in Col. 5 instead, the density range would have been 5300 to $60400 \mathrm{~cm}^{-3}$ instead of 820 to $16900 \mathrm{~cm}^{-3}$.

We find velocity gradients in $\mathrm{CS}$ and $\mathrm{C}^{18} \mathrm{O}$ towards five of the mapped clouds. Position-velocity plots from Gaussian fits along the direction showing the gradient are shown in Fig. 8. Least squares fits to these data gave the velocity gradient in Col. 10 of Table 5 . It appears that the gradient in the CS cloud near WB89 1187 is due to three clumps with a constant velocity each. For comparison with the assumed $\mathrm{C}^{18} \mathrm{O}$ excitation temperature $(20 \mathrm{~K})$ we list in Col. 11 the excitation temperature derived from the maximum ${ }^{12} \mathrm{CO}(1-0) T_{\mathrm{A}}^{*}$ in the cloud at the offset position in Col. 12. The value in the cloud center for $\mathrm{C}^{18} \mathrm{O}$ will be higher or lower, depending on whether there are heating sources or not. $\mathrm{NH}_{3}$ observations of similar clouds showed that the kinetic temperature also depends on the luminosity of the embedded source (see e.g. Wouterloot et al. 1988).

\section{Comments on individual sources}

WB89 1086: The Digital Sky Survey image shows Bran 96 as a slightly elongated ( $50^{\prime \prime}$ approximately in the east-west direction) nebulosity centered at the PSC position. Some obscuration might be discernible $30^{\prime \prime}$ north of this position where we find the weak $\mathrm{C}^{18} \mathrm{O}$ maximum in Fig. 6 . Also the CS emission is weak. Northeast of the PSC position there is a $4 \sigma$ detection which seems real and is visible as the strongest (CS) peak in Fig. 6. WB89 1086 shows only emission centered at $62-64 \mathrm{~km} \mathrm{~s}^{-1}$, which is associated with the PSC source. Although the spectrum taken by WB89 suggested the presence of broad wing emission, this is not confirmed by the present data.

WB89 1099: The velocity of the main component is about $5.5 \mathrm{~km} \mathrm{~s}^{-1}$. Emission at higher velocities is detected SE of the PSC position and NW of the PSC position (9.5$\left.10 \mathrm{~km} \mathrm{~s}^{-1}\right)$. In addition, at most positions, except SE of the PSC position, we find emission at $3.5-4.5 \mathrm{~km} \mathrm{~s}^{-1}$, which close to the PSC position is visible only as a shoulder of the main component, and difficult to separate from it. At most positions there is also very weak $(<1 \mathrm{~K})$ emission at about $-1 \mathrm{kms}^{-1}$, which is too faint to derive its distribution. ${ }^{12} \mathrm{CO}$ emission towards this source has been mapped with the SEST also by Olberg et al. (1989) and Nielsen et al. (1998). The latter authors in addition mapped ${ }^{13} \mathrm{CO}$, and observed some positions in $\mathrm{C}^{18} \mathrm{O}$, and CS. $\mathrm{CS}(2-1), \mathrm{CO}(2-1)$, and $1.3 \mathrm{~mm}$ continuum emission were observed towards this object (DC 253.3-1.6) by Henning \& Launhardt (1998). We note that Nielsen et al. (1998) consider the $4 \mathrm{~km} \mathrm{~s}^{-1}$ component to be associated to the main line at $5.5 \mathrm{~km} \mathrm{~s}^{-1}$ (in their Fig. 7). However this component was also detected in ${ }^{13} \mathrm{CO}$ and appears to be stronger at larger distances from the PSC position. It might be gas moving away from the main part of the globule CG 30 (but which is still associated with it). HH 120 is located $5^{\prime \prime}$ north of the PSC position, close to the southern maximum of $T_{\mathrm{A}}^{*}$ (see Fig. 3) (the northern peak is slightly stronger: $12.1 \mathrm{~K}$ vs. $11.8 \mathrm{~K}$ ). The distribution of blue and red outflow emission in Fig. 4 agrees with the results of Olberg et al. (1989) and Nielsen et al. (1998), with a stronger red wing east of the PSC position and a weaker blue wing west of it. The mass that we derived is slightly higher than the one by Nielsen et al. (1998) (after correcting for the different adopted distance) because these authors assumed that the gas is optically thin. The 
Table 4. $\mathrm{CS}$ and $\mathrm{C}^{18} \mathrm{O}$ results. The first line for each source gives the parameters of the averaged cloud spectrum, the second line those at the peak $T_{\mathrm{A}}^{*}$ position

\begin{tabular}{|c|c|c|c|c|c|c|c|c|c|}
\hline \multirow{2}{*}{$\begin{array}{r}\text { Source } \\
\text { WB89 } \\
\\
(1) \\
\end{array}$} & \multirow[b]{2}{*}{$(2)$} & \multicolumn{4}{|c|}{ CS } & \multicolumn{4}{|c|}{$\mathrm{C}^{18} \mathrm{O}$} \\
\hline & & $\begin{array}{c}T_{\mathrm{A}}^{*} \\
\mathrm{~K} \\
(3) \\
\end{array}$ & $\begin{array}{c}\text { offset } \\
\prime \prime \\
(4) \\
\end{array}$ & $\begin{array}{c}V_{\mathrm{lsr}} \\
\mathrm{km} \mathrm{s}^{-1} \\
(5) \\
\end{array}$ & $\begin{array}{c}\Delta v \\
\mathrm{kms}^{-1} \\
(6)\end{array}$ & $\begin{array}{l}T_{\mathrm{A}}^{*} \\
\mathrm{~K} \\
(7) \\
\end{array}$ & $\begin{array}{c}\text { offset } \\
\text { " } \\
(8) \\
\end{array}$ & $\begin{array}{c}V_{\mathrm{lsr}} \\
\mathrm{km} \mathrm{s}^{-1} \\
(9)\end{array}$ & $\begin{array}{c}\Delta v \\
\mathrm{kms}^{-1} \\
(10)\end{array}$ \\
\hline \multirow[t]{2}{*}{1086} & aver. & $0.12(0.02)$ & & $63.84(0.08)$ & $1.12(0.16)$ & $0.16(0.02)$ & & $63.63(0.07)$ & $1.63(0.17)$ \\
\hline & peak & $0.57(0.08)$ & $(-40,-80)$ & $63.89(0.07)$ & $0.60(0.13)$ & $0.44(0.02)$ & $(0,0)$ & $63.88(0.03)$ & $1.03(0.06)$ \\
\hline \multirow[t]{2}{*}{1099} & aver. & $0.52(0.03)$ & & $5.85(0.02)$ & $1.12(0.06)$ & $0.45(0.03)$ & & $5.81(0.02)$ & $0.94(0.05)$ \\
\hline & peak & $2.33(0.18)$ & $(0,0)$ & $6.06(0.01)$ & $1.34(0.03)$ & $1.05(0.05)$ & $(0,0)$ & $6.04(0.01)$ & $0.93(0.03)$ \\
\hline \multirow[t]{2}{*}{1135} & aver. & $0.36(0.03)$ & & $54.66(0.05)$ & $2.07(0.12)$ & $0.26(0.03)$ & & $54.53(0.05)$ & $1.77(0.14)$ \\
\hline & peak & $1.05(0.07)$ & $(0,0)$ & $54.83(0.03)$ & $2.88(0.09)$ & $0.45(0.06)$ & $(0,0)$ & $55.13(0.05)$ & $3.34(0.13)$ \\
\hline \multirow[t]{2}{*}{1173} & aver. & $0.59(0.02)$ & & $7.37(0.02)$ & $1.24(0.05)$ & $0.57(0.04)$ & & $7.36(0.01)$ & $1.31(0.03)$ \\
\hline & peak & $1.53(0.16)$ & $(40,-40)$ & $7.20(0.04)$ & $1.17(0.09)$ & $1.45(0.13)$ & $(-40,0)$ & $7.25(0.02)$ & $0.99(0.08)$ \\
\hline \multirow[t]{2}{*}{1181} & aver. & $1.15(0.05)$ & & $4.01(0.02)$ & $3.47(0.04)$ & $0.36(0.02)$ & & $4.20(0.02)$ & $2.95(0.05)$ \\
\hline & peak & $4.15(0.20)$ & $(0,0)$ & $4.08(0.01)$ & $4.03(0.03)$ & $1.00(0.11)$ & $(-40,0)$ & $3.45(0.05)$ & $3.08(0.12)$ \\
\hline \multirow[t]{2}{*}{1187} & aver. & $0.53(0.03)$ & & $12.19(0.02)$ & $2.79(0.04)$ & $0.31(0.03)$ & & $12.06(0.04)$ & $2.55(0.10)$ \\
\hline & peak & $1.25(0.20)$ & $(40,0)$ & $12.97(0.08)$ & $2.20(0.13)$ & $0.72(0.12)$ & $(40,0)$ & $12.58(0.07)$ & $1.83(0.18)$ \\
\hline \multirow[t]{2}{*}{1189} & aver. & $0.36(0.03)$ & & $2.47(0.03)$ & $1.37(0.08)$ & $0.41(0.02)$ & & $2.44(0.02)$ & $1.26(0.05)$ \\
\hline & peak & $1.61(0.20)$ & $(40,0)$ & $2.31(0.04)$ & $1.56(0.09)$ & $1.06(0.11)$ & $(40,0)$ & $2.35(0.03)$ & $1.02(0.07)$ \\
\hline \multirow[t]{2}{*}{1262} & aver. & $0.77(0.04)$ & & $9.50(0.02)$ & $2.57(0.05)$ & \multirow{2}{*}{\multicolumn{4}{|c|}{ not observed }} \\
\hline & peak & $3.75(0.26)$ & $(0,0)$ & $9.38(0.03)$ & $3.15(0.08)$ & & & & \\
\hline
\end{tabular}

Table 5. Properties of clumps mapped in $\mathrm{CS}$ and $\mathrm{C}^{18} \mathrm{O}$

\begin{tabular}{|c|c|c|c|c|c|c|c|c|c|c|c|}
\hline \multirow{3}{*}{$\begin{array}{r}\text { Source } \\
\text { WB89 } \\
\\
(1)\end{array}$} & \multicolumn{4}{|c|}{$\begin{array}{c}r \\
\mathrm{pc}\end{array}$} & \multicolumn{2}{|c|}{$\begin{array}{c}M_{\text {vir }} \\
M_{\odot}\end{array}$} & \multirow{2}{*}{\multicolumn{2}{|c|}{$\begin{array}{c}M_{\mathrm{LTE}} \\
M_{\odot} \mathrm{cm}^{-3} \\
\mathrm{C}^{18} \mathrm{O}\end{array}$}} & \multirow{3}{*}{$\begin{array}{c}\mathrm{d} v / \mathrm{d} r \\
\mathrm{~km} \mathrm{~s}^{-1} \mathrm{pc}^{-1} \\
(10)\end{array}$} & \multirow{3}{*}{$\begin{array}{l}T_{\text {ex }} \\
\mathrm{K} \\
(11) \\
\end{array}$} & \multirow{3}{*}{\begin{tabular}{ll} 
& offset \\
& \multicolumn{1}{l}{${ }^{12} \mathrm{CO}$} \\
& \\
& $(12)$ \\
\end{tabular}} \\
\hline & \multicolumn{2}{|c|}{$\mathrm{CS}$} & \multicolumn{2}{|c|}{$\mathrm{C}^{18} \mathrm{O}$} & $\mathrm{CS}$ & $\mathrm{C}^{18} \mathrm{O}$ & & & & & \\
\hline & $(2)$ & (3) & (4) & $(5)$ & (6) & $(7)$ & (8) & (9) & & & \\
\hline 1086 & 1.4 & $<0.9^{1}$ & 1.2 & 0.70 & 260 & 480 & 500 & 1000 & & 23.0 & $(-24,-6)$ \\
\hline 1099 & 0.29 & 0.10 & 0.24 & 0.16 & 47 & 29 & 25 & 6500 & $+1.51(0.23)$ & 15.5 & $(-15,16)$ \\
\hline 1135 & 1.5 & 0.69 & 2.1 & 1.1 & 920 & 830 & 2120 & 820 & $-0.40(0.08)$ & 16.1 & $(-2,-11)$ \\
\hline 1173 & $>0.76$ & $>0.39^{2}$ & 1.1 & 0.72 & $>150$ & 240 & 6300 & 16900 & & 41.1 & $(-42,46)$ \\
\hline 1181 & 0.50 & 0.17 & 0.56 & 0.30 & 770 & 625 & 260 & 5300 & $-2.01(0.39)$ & 29.3 & $(6,19)$ \\
\hline 1187 & 1.8 & 1.1 & $>0.81$ & $>0.68$ & 1770 & $>700$ & $>470$ & 3200 & $+0.42(0.04)$ & 23.2 & $(17,3)$ \\
\hline 1189 & 0.19 & 0.09 & 0.17 & 0.11 & 47 & 36 & 17 & 12400 & $+1.91(0.20)$ & 29.0 & $(13,19)$ \\
\hline 1262 & 1.6 & 0.47 & & & 1350 & & & & & & \\
\hline 1275 & & & & & & & & & & 28.4 & $(47,24)$ \\
\hline
\end{tabular}

1 Two unresolved clumps.

${ }^{2}$ The main clump; there are also two unresolved $(<0.18 \mathrm{pc})$ clumps.

other parameters show larger differences due to the different methods used for calculating them.

WB89 1135: There is some foreground emission at about $44 \mathrm{~km} \mathrm{~s}^{-1}$, mainly to the SE of the PSC position, but it can be clearly separated from the cloud emission, which is at about $54 \mathrm{~km} \mathrm{~s}^{-1}$. The source is located near the south eastern edge of a more extended cloud (the highest $T_{\mathrm{A}}^{*}$ value of $9.8 \mathrm{~K}$ is found in our map at offset $\left(-80^{\prime \prime}, 60^{\prime \prime}\right)$ ). No optical emission is visible towards this source on the Digital Sky Survey image.

WB89 1173: This source is associated with the bright rimmed cloud 55 found on the ESO R print by Sugitani
\& Ogura (1994). The rim approximately follows the lowest contours in Fig. 3. The relatively high temperature $\left(T_{\mathrm{A}}^{*}=26 \mathrm{~K}\right.$, i.e. $\left.T_{\mathrm{ex}}=41 \mathrm{~K}\right)$ found north-west of the IRAS PSC position (higher than towards any other source in the sample) might be related to the presence of this bright rim, and caused by the same heating source. However, the $T_{\mathrm{A}}^{*}$ maximum is located at the position of some nebulosity visible at the Digital Sky Survey image, which does not show the rim. Liseau et al. (1992) and Massi et al. (1999) found only relatively weak NIR emission towards WB89 1173 (their source IRS 63). Massi et al. (1997) detected jetlike $\mathrm{H}_{2}$ emission towards this source. Combined with the fact that we detect strong outflow emission, these data 
suggest that the embedded IRAS source is very young. Emission at velocities different from the main component $\left(7-7.5 \mathrm{~km} \mathrm{~s}^{-1}\right)$ is found at $8.5-9.2 \mathrm{~km} \mathrm{~s}^{-1}$ ( $\mathrm{N}$ and $\mathrm{W}$ of the PSC position), and at $4-4.5 \mathrm{~km} \mathrm{~s}^{-1}$ (SE of the PSC position). Both components have small line widths $\left(1 \mathrm{~km} \mathrm{~s}^{-1}\right)$.

WB89 1181: This region shows only emission centered at $3-4 \mathrm{~km} \mathrm{~s}^{-1}$, which is associated with the PSC source. Liseau et al. (1992) and Massi et al. (1999) detected strong NIR emission towards this source (their IRS 17), which shows no optical emission on the Digital Sky Survey (DSS) image. Massi et al. (1997) detected an $\mathrm{H}_{2}$ jet, about $1^{\prime}$ in size towards IRS 17 . On the DSS there are two stars with associated nebulosity at offsets $\left(40^{\prime \prime}, 100^{\prime \prime}\right)$ and $\left(22^{\prime \prime}\right.$, $\left.138^{\prime \prime}\right)$ from the PSC position, which might be associated with the $\mathrm{CO}$ cloud. They are located in a $T_{\mathrm{A}}^{*}$ minimum in Fig. 3.

WB89 1187: In addition to the main component near $13 \mathrm{~km} \mathrm{~s}^{-1}$, most spectra away from the centre show an additional narrower line at $9-10.3 \mathrm{~km} \mathrm{~s}^{-1}$. Near the PSC position, a comparison of ${ }^{12} \mathrm{CO}$ and ${ }^{13} \mathrm{CO}$ spectra suggests the presence of self-absorption (the ${ }^{13} \mathrm{CO}$ maximum and a ${ }^{12} \mathrm{CO}$ dip are at the same velocity). However, considering all positions it is more likely that there are really two components everywhere and the single (but broad) ${ }^{13} \mathrm{CO}$ peak is caused by two lines that are close in velocity. At two positions there is also emission at about $5 \mathrm{~km} \mathrm{~s}^{-1}$. This source is associated with the NIR source IRS 19 (Liseau et al. 1992; Massi et al. 1999) and is optically invisible. Very weak $\mathrm{H}_{2}$ emission was found about $1^{\prime}$ east of the PSC position (Massi et al. 1997).

WB89 1189: The velocity of the peak CO emission near the PSC position is about $1.5 \mathrm{~km} \mathrm{~s}^{-1}$, whereas at (positive and negative) offsets in $\delta$, velocities are about $3.3 \mathrm{~km} \mathrm{~s}^{-1}$. We assume that this is the same component, showing a velocity gradient (see also Fig. 8, where there is a similar, but smaller gradient). From the line profiles we conclude that NW of the PSC position there is an additional component at $3.6-4 \mathrm{~km} \mathrm{~s}^{-1}$. Other components are found at $6-7.5 \mathrm{~km} \mathrm{~s}^{-1}$ and at $12.5-13.5 \mathrm{~km} \mathrm{~s}^{-1}$. NIR emission was detected towards WB89 1189 by Liseau et al. (1992) and Massi et al. (1999) (source IRS 20). This source is optically invisible. A small bipolar $\mathrm{H}_{2}$ jet (extent about $5^{\prime \prime}$ towards both sides of a central object) was found by Massi et al. (1997), and is located north of the CS and $\mathrm{C}^{18} \mathrm{O}$ clumps.

WB89 1262: This source ( $\mathrm{G} 270.26+0.83)$ was observed in several molecular transitions by Zinchenko et al. (1995) and Lapinov et al. (1998). Our CS(2-1) map looks similar to the one from those authors, however we find that the maximum is close to the IRAS PSC position rather than near $\left(-30^{\prime \prime}, 30^{\prime \prime}\right)$.

WB89 1275: The optical image of RCW 42 shows a dust lane which is coincident with the distribution of peak $T_{\mathrm{A}}^{*}$ of ${ }^{12} \mathrm{CO}$ in Fig. 3. There are no radio continuum maps available which would show whether this dust lane is seen in projection towards RCW 42, or actually divides the region into two parts. In this source the $\mathrm{CO}$ maximum is displaced from the PSC position to the NE. The strongest component is at $36-38 \mathrm{~km} \mathrm{~s}^{-1}$, whereas another one is visible at most positions at $39-46 \mathrm{~km} \mathrm{~s}^{-1}$, which is difficult to separate. A third but weak component is at about $28-34 \mathrm{~km} \mathrm{~s}^{-1}$ in the western part of the mapped region. At two positions south of the source there is emission at $20 \mathrm{~km} \mathrm{~s}^{-1}$. The broad line profiles in the ${ }^{12} \mathrm{CO}$ map are interpreted as the superposition of two separate emission profiles, both without signs of outflow. The secondary $\left(39-46 \mathrm{~km} \mathrm{~s}^{-1}\right)$ velocity component has its maximum south of the dust lane where there is optical emission, and might be located behind RCW 42 .

\section{Conclusions}

We present maps of the molecular emission towards nine IRAS sources, which in the WB89 catalogue had distinctly broad wings in their line profiles. Observations were done with the SEST, at the frequencies of ${ }^{12} \mathrm{CO}(1-0),{ }^{13} \mathrm{CO}(1-$ $0), \mathrm{C}^{18} \mathrm{O}(1-0)$ and $\mathrm{CS}(2-1)$. From our ${ }^{12} \mathrm{CO}(1-0)$ maps we conclude that seven of those sources (WB89 1099, 1135, $1173,1181,1187,1189,1275)$ are genuine outflow sources. One source (WB89 1275) shows a blue wing, but possible red wing emission is confused due to a close second velocity component. WB89 1086 shows no outflow emission at all. The remaining source (WB89 1262) was only observed in CS. The sources are all in or behind the region of the Vela Molecular Ridge (VMR), an active star-forming region, consisting of at least 4 GMC's with distances between 0.5 and $2-3 \mathrm{kpc}$. Based on the distances and the line velocities (Table 1 ), at most 6 of our sources are actually associated with the VMR; the remaining 3 lie at least $4 \mathrm{kpc}$ further away.

The "pure" outflow emission was found by first subtracting the contribution to the emission of non-associated components, using Gaussian fits. These additional emission lines were identified at positions away from the center of the flows. Then a correction was applied for the contribution of the emission of the quiescent gas to the wing emission (Sect. 3.1). Parameters of the outflow gas were then derived, assuming $T_{\mathrm{ex}}=20 \mathrm{~K}$ and $\tau=1$ in all cases. All but two of the detected outflows are associated with IRAS sources located in the VMR (WB89 1135 and 1262 are more distant). The total (blue + red) velocity range of the flows is between 15.4 and $25.9 \mathrm{~km} \mathrm{~s}^{-1}$. The outflows are barely resolved in the present observations: the spatial extent $(0.22-2.65 \mathrm{pc})$ is about twice the beam size (in pc) in all sources. The mass contained in the flows lies between $1.1 M_{\odot}(\mathrm{WB} 891099)$ and $150 M_{\odot}$ (WB89 1135), which are (perhaps not surprisingly) the nearest and farthest objects where an outflow has been detected. Likewise, these two sources also determine the maximum range in energy 
$\left(0.6-16110^{44} \mathrm{erg}\right)$. The dynamical timescale of all flows is of the order of $10^{5}$ years, typical for the age of Class I sources.

$\mathrm{C}^{18} \mathrm{O}(1-0)$ emission was observed and detected towards 7 out of 9 objects; $\operatorname{CS}(2-1)$ towards 8 out of 9 . All spectra show single emission components, three of them show also line wings in CS emission. Single clumps are found in 5 sources, two sources have several clumps in one of the transitions (the other one not being mapped completely), and one source shows only weak emission. Masses range from 10 to $1000 M_{\odot}$, radii from 0.2 to 2 pc. Radii determined from $\mathrm{C}^{18} \mathrm{O}$ and $\mathrm{CS}$ are similar. A velocity gradient $\left(-2.0\right.$ to $\left.+1.9 \mathrm{~km} \mathrm{~s}^{-1} \mathrm{pc}^{-1}\right)$ is found in 5 sources.

Acknowledgements. This research was partly supported by the Deutsche Forschungsgemeinschaft through grant SFB-301. We thank C. Kramer and F. Massi for their comments on an earlier version of this paper. This research has made use of the SIMBAD database, operated at CDS, Strasbourg, France.

\section{References}

Bachiller R., 1996, ARA\&A 34, 111

Bronfman L., Nyman L.-Å., May J., 1996, A\&AS 115, 81

Brand J., Blitz L., 1993, A\&A 275, 67

Cabrit S., Bertout C., 1990, ApJ 348, 530

DeYoung D.S., 1986, ApJ 307, 62

Frerking M.A., Langer W.D., Wilson R.W., 1982, ApJ 262, 590

Fukui Y., Iwata T., Mizuno A., Bally J., Lane A.P., 1993, Molecular outflows, in: Levine E.H., Lunine J.I. (eds.), Protostars and Planets III, p. 603

Henning Th., Launhardt R., 1998, A\&A 338, 242

Lada C.J., 1985, ARA\&A 23, 267

Lapinov A.V., Schilke P., Juvela M., Zinchenko I.I., 1998, A\&A 336, 1007

Liseau R., Lorenzetti D., Nisini B., Spinoglio L., Moneti A.,
1992, A\&A 265, 577

MacLaren I., Richardson K.M., Wolfendale A.W., 1988, ApJ 333,821

Massi F., 1999, Ph.D. Thesis, Universitá di Roma "La Sapienza"

Massi F., Lorenzetti D., Vitali F., 1997, Near Infrared $\mathrm{H}_{2}$ imaging of YSO's in Vela Molecular Clouds, in: Malbet F., Castets A. (eds.), IAU 182 Symposium Poster Proceedings. Observatoire de Grenoble, p. 21

Massi F., Giannini T., Lorenzetti D., et al., 1999, A\&AS 136, 471

Murphy D.C., May J., 1991, A\&A 247, 202

Nielsen A.S., Olberg M., Knude J., Booth R.S., 1998, A\&A 336,329

Olberg M., Reipurth B., Booth R.S., 1989, in: Winnewisser G., Armstrong J.T. (eds.). The Physics and Chemistry of Interstellar Molecular Clouds, p. 120

Shepherd D.S., Churchwell E., 1996, ApJ 472, 225

Shu F.H., Ruden S.P., Lada C.J., Lizano S., 1991, ApJ 370, L31

Snell R.L., Scoville N.Z., Sanders D.B., Erickson N.R., 1984, ApJ 284, 176

Sugitani K., Ogura K., 1994, ApJS 92, 163

Tafalla M., Bachiller R., Wright M.C.H., Welch W.J., 1997, ApJ 474, 329

Wilson T.L., Rood R., 1994, ARA\&A 32, 191

Wilson T.L., Serabyn E., Henkel C., Walmsley C.M., 1986, A\&A 158, L1

Wouterloot J.G.A., Brand J., 1989, A\&AS 80, 149 (WB89)

Wouterloot J.G.A., Walmsley C.M., Henkel C., 1988, A\&A 203, 367

Wouterloot J.G.A., Henkel C., Walmsley C.M., 1989, A\&A 215,131

Wouterloot J.G.A., Brand J., Burton W.B., Kwee K.K., 1990, A\&A 230, 21

Zinchenko I., Mattila K., Toriseva M., 1995, A\&AS 111, 95 